\title{
Signal Analysis of Optical Interference in Relation to Colorimetry for Measurements Made Along Individual Myofibers in Cooked Beef
}

\author{
Howard J. Swatland \\ Department of Animal Biosciences, \\ University of Guelph, \\ Guelph, Ontario, Canada \\ Email: Swatland [AT] uoguelph.ca
}

\begin{abstract}
Subsurface reflective interference from A-bands in roast beef persisted under water and some myofibers had interference peaks exceeding the reflectance of white barium sulfate. The number of interference peaks was correlated with CIE (Commission Internationale de l'Éclairage) Y\% $(r=0.51, P<0.001)$. As the number of peaks increased, the distance from the central white of the CIE chart decreased $(r=-0.52, P<0.001)$. Myofibers with low scattering had fewer interference peaks $(2.9 \pm 0.3, n=10)$ than myofibers with high scattering $(4.9 \pm 1.3, n=31, P<$ 0.001 . Thus, the number of reflecting and interfering layers may be important in relating light scattering along myofibers to surface iridescence. One or a few reflective layers may produce strong interference colors while many layers may produce colorless scattering.
\end{abstract}

\section{INTRODUCTION}

Light scattering has a strong effect on the colorimetry of a variety of complex red structures including meat, where the dominant chromophore is myoglobin and its derivatives [1]. There are differences in light scattering along versus across myofibers, so that color measurements made across myofibers may differ from color measurements made along myofibers [2]. Light scattering across the long axes of myofibers is strongly affected by meat $p \mathrm{H}$. X-ray diffraction shows that the lateral spacing of myofilaments depends on $p \mathrm{H}$ [3] and this changes the refractive indices of myofibrils [4]. Thus, a low $p \mathrm{H}$ causes tightly packed myofilaments, an increase in refractive index, and an increase in light scattering.

The situation for light passing along the long axes of myofibers is less well known. Light scattering along myofibers is sometimes augmented by multilayer reflective interference from myofibrillar A-bands causing iridescence [5]. Is scattering related to iridescence? Signal processing of interference spectra correlated with colorimetry by the weighted ordinate method gave an interesting answer.

\section{MATERIALS AND METHODS}

\subsection{Colorimetry}

The weighted-ordinate calculation for color coordinates in the CIE system was taken from Billmeyer and Saltzman [6]. The hypotenuse in color $x-y$ space from the central white at $0.33 x$ and $0.33 y$ is a measure of chromatic intensity. With the Pythagoras theorem, all results are positive in sign.

Hypotenuse $=\left((0.33-x)^{2}+(0.33-y)^{2}\right)^{0.5}$

In other words, $0.33 x$ and $0.33 y$ was used as a measure of colorless scattering, while the length of the hypotenuse into the color space was used as a measure of iridescence. Extensive testing has shown that the weighted-ordinate method may be used for light microscopy with negligible complications from different corrections for chromatic aberration and illuminator emission spectra, provided that the area to be measured uniformly fills the photometer aperture [7]. 


\subsection{Signal processing}

Working in sequence through the spectrum from 400 to $700 \mathrm{~nm}$, a flag was maintained for the direction of change (up or down). When reflectance was constant the flag remained unchanged. The start of a new peak was taken as the point at which the flag changed from down to up. The tip of a peak was taken as the point at which the flag changed from up to down. At the start of a new peak, the wavelength was stored. At the tip of a peak, the reflectance intensity was stored for the later calculation of mean peak intensity, and the distance from the start to the tip of the peak was stored for the later calculation of mean half peak width. Any asymmetry of peaks was cancelled in the determination of a mean value for half peak width, provided that the direction of asymmetrical peaks was random (as it appeared to be on subjective evaluation of graphs of the data). When peaks overlapped, peak intensity was recorded separately for each peak, and half peak width was shared for all except the first peak of a cluster. The measurement of half peak width rather than full peak width simplified the algorithm for the detection of peaks separated by flat bottomed valleys. The half peak width of a reflectance peak could be found with relatively few pointers. One pointer recorded where values started to rise and another recorded reflectance at the last position measured. Having moved past the tip of a peak, incoming values began to decrease and the half peak width (from the start to the tip) was found retrospectively.

\subsection{Microscopy}

A Zeiss Universal microscope (Carl Zeiss, D-7082 Oberkochen, Germany) was fitted with accessories for reflectance microscopy (identified by a Zeiss part number). The microscope was operated from a type MPC 64 control module (Zeiss 477469) on a bus (IEEE 488). The photomultiplier was a side-window Hamamatsu (1126 Ichino-Cho, Hamamatsu City, Japan) HTV R 928 with S-20 characteristics operated as described in Zeiss bulletin G-41-9000.1/11-e. A grating monochromator (Zeiss 474345) with stray-light filters (Zeiss 477215) was mounted under the photomultiplier and scanned from 400 to $700 \mathrm{~nm}$ in steps of $10 \mathrm{~nm}$ with a $10 \mathrm{~nm}$ band-pass. Illumination was from a $100 \mathrm{~W}$ halogen source with a stabilized power supply (Hewlett-Packard 6642A) directed through a solenoid shutter and into a vertical illuminator (Zeiss II-C with H-PL-POL beam splitter). The photometer head was a type 03 (Zeiss 477304). The microscope objectives were long-distance Epiplan for reflected light (no cover slip). To allow for a depth of water over samples, the objective was $\times$ 4 , NA 0.01 . Other measurements were made with $\times 16$, NA 0.30 . The system was standardized on optical quality white barium sulfate powder at fractions of the dynamic range of the photomultiplier, knowing that constructive interference might exceed the white reflectance standard.

\subsection{Samples}

The iridescent beef was from iliocostalis muscles of prime rib roasts (dry aged for several weeks and lightly roasted). The myofibers measured $(n=63)$ originated from eight carcasses (Canada Grade AAA). Samples were cut into approximately $1-\mathrm{mm}$ thick slices, and $1-\mathrm{cm}$ diameter discs were cut with a cork borer. For several samples, discs were mounted in ring slides so that the ring was slightly higher then the meat disc. This allowed distilled water to be added to create a concave meniscus, avoiding direct reflectance from the water surface and allowing iridescent meat to be viewed under water. Thus, the data reported here are from subsurface multilayer reflectance not from surface diffraction.

\section{RESULTS AND DISCUSSION}

Before starting on the main analysis of iridescent myofibers, it is appropriate to show results from non-iridescent myofibers. Meat color is normally measured macroscopically with several square centimeters illuminated so that diffuse reflectance involves a considerable subsurface light path through the meat for myoglobin absorbance. When working with an optical path along an individual myofiber, a typical meat spectrum with strong myoglobin absorbance was created when the light was transmitted along the myofiber (illumination from below, spectrophotometry from above), as in the transmittance spectrum shown in Figure 1. Figure 1 shows a typical myoglobin spectrum with a dimple around $570 \mathrm{~nm}$ and strong absorbance of blue and green light. 


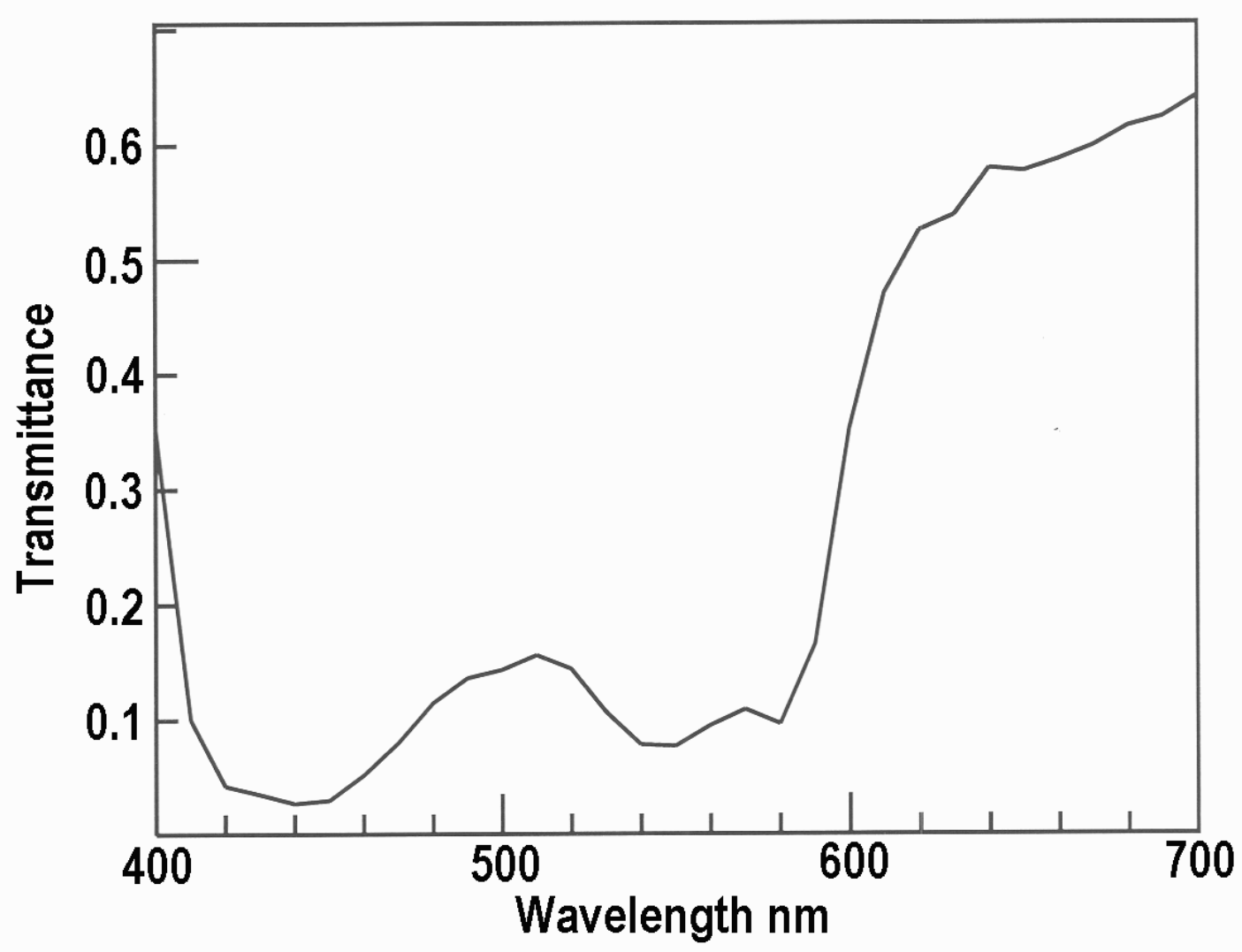

Figure 1. Transmittance spectrum along the length of a myofiber with minimal heat denaturation in a beef roast.

But when illumination and spectrophotometry were both above the myofiber, the reflectance spectrum from the same myofiber was completely different (Figure 2). Specular reflectance from part of the myofiber had a fairly flat spectrum with greater intensity at $400 \mathrm{~nm}$ than at $700 \mathrm{~nm}$, a typical consequence of scattering being inversely proportional to wavelength.[8] This was also evident in the diffuse spectrum, but now myoglobin absorbance around $550 \mathrm{~nm}$ gave a broad valley (Figure 2B). 


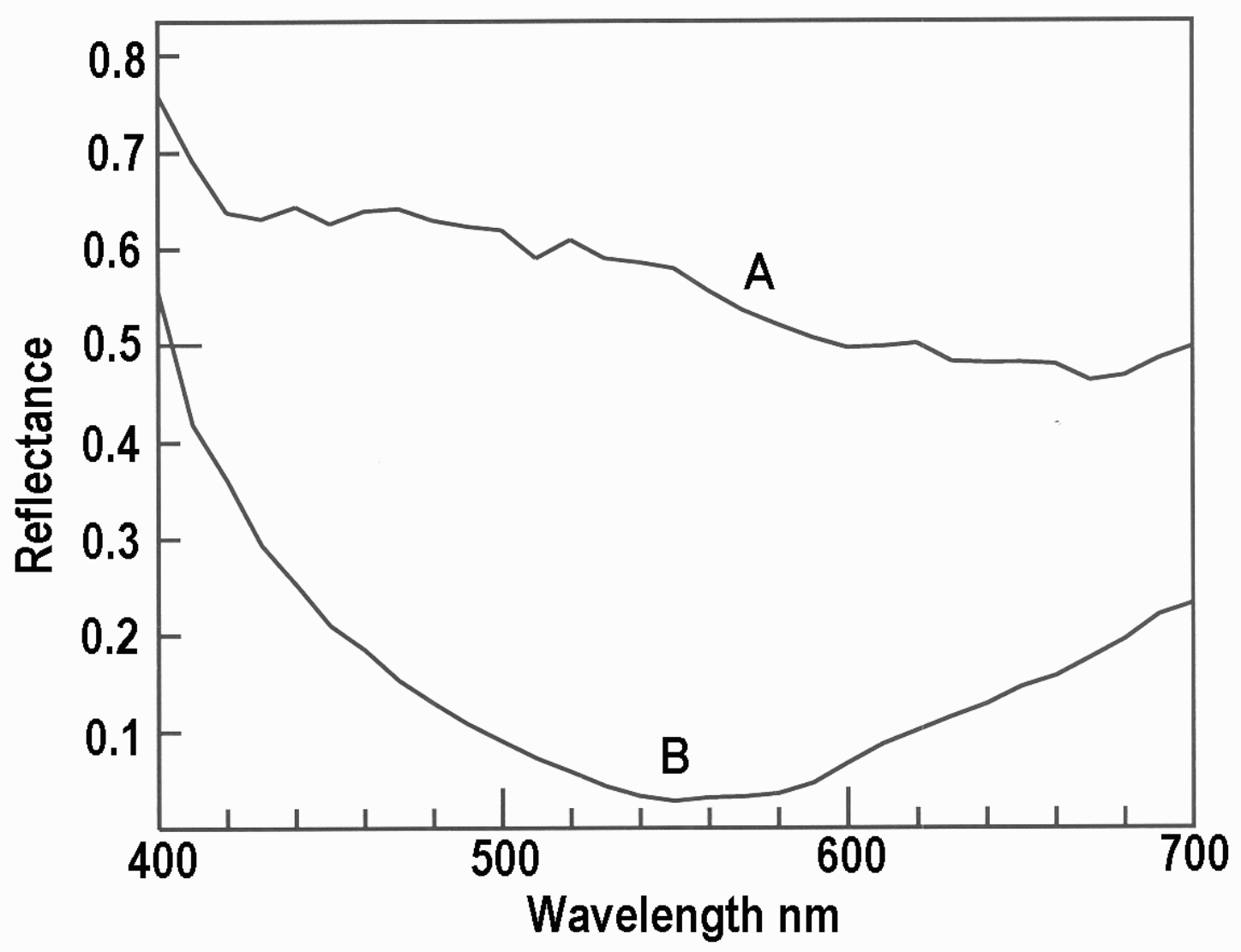

Figure 2. Specular reflectance spectrum (line A) and diffuse reflectance spectrum (line B) from parts of the same myofiber shown in Figure 1.

The reflectance spectra of iridescent myofibers differed from the spectra of non-iridescent myofibers just described, although there were some similarities (compare line B in Figure 2 with line B in Figure 3). The spectra of iridescent myofibers were dominated by interference peaks (Figure 3). With constructive interference it was possible for peaks to exceed the reflectance of white barium sulfate (Figure $3 \mathrm{~A}$, peak 3 ). 


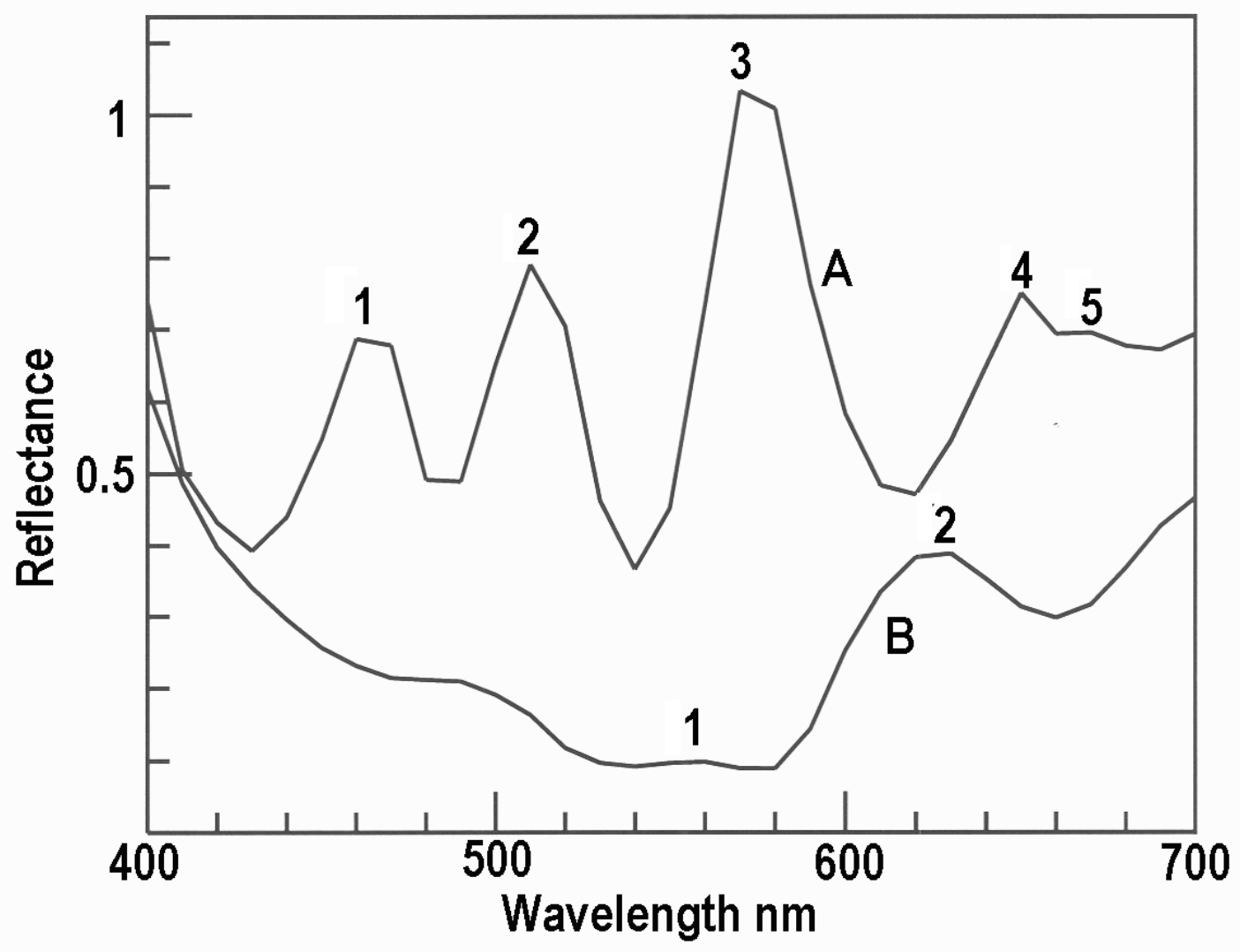

Figure 3. Reflectance spectra of iridescent myofibers with five (line A) or two peaks (line B) as counted by the signal processing algorithm. Note descending peaks starting at $400 \mathrm{~nm}$ and uncompleted peaks at $700 \mathrm{~nm}$ were not counted and peaks might start with only a very small increase in reflectance (A5 and B1).

The color coordinates of three iridescent myofibers with a strong metallic appearance are shown in Figure 4. Their distance from the central white area at $x=0.33$ and $y=0.33$ was calculated by the Pythagoras theorem. 


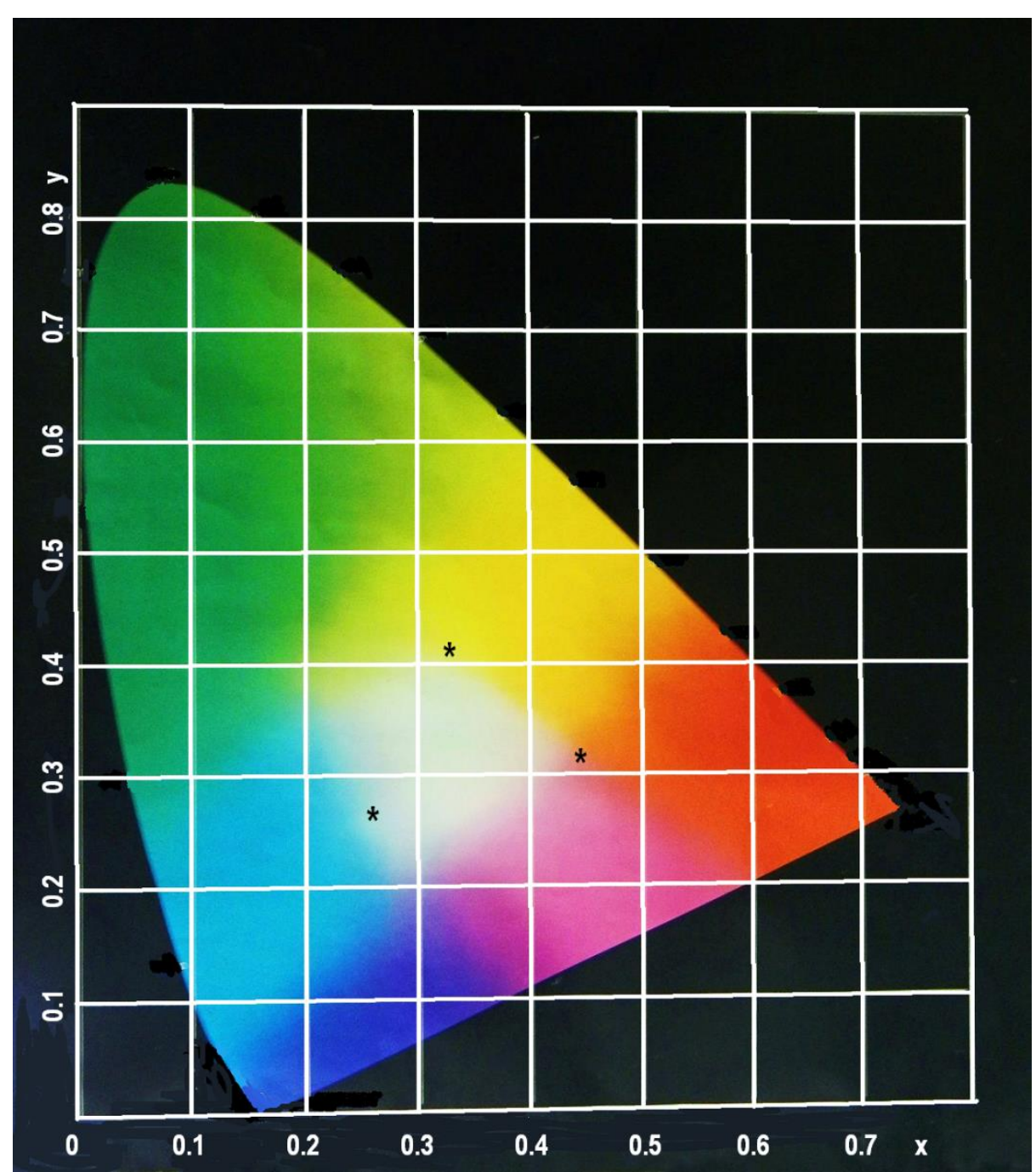

Figure 4. The CIE color space showing coordinates for three myofibers $(*)$ with a strong metallic appearance.

Table 1. Mean values for signal analysis and color coordinates

\begin{tabular}{lll}
\hline & Mean & SD \\
\hline Number of peaks & 4.38 & 1.39 \\
Mean peak intensity & 0.30 & 0.12 \\
Mean peak width nm & 45.48 & 15.14 \\
Peak 1 intensity & 0.61 & 0.35 \\
Peak 2 intensity & 0.26 & 0.12 \\
& & \\
Peak 3 intensity & 0.20 & 0.11 \\
CIE $x$ & 0.38 & 0.04 \\
CIE $y$ & 0.31 & 0.03 \\
CIE \%Y & 19.24 & 6.07 \\
Distance from white & 0.07 & 0.02 \\
\hline
\end{tabular}

The number of interference peaks had only a weak relationship with CIE $x(r=-0.20$, NS) but a stronger relationship with CIE $y \quad(r=0.43, P<0.001)$. All statistical tests had 2-tails and $\mathrm{n}=63$. The number of interference peaks was correlated with CIE Y\% $(r=0.51, P<0.001)$. As the number of peaks increased, the distance from central white decreased $(r=-0.52, P<0.001)$. Thus, myofibers with a strong metallic iridescence tended to have one or two strong interference peaks while myofibers with numerous interference peaks approached the central white area.

The mean intensity of interference peaks was weakly correlated with CIE $x(r=0.25, P<0.05)$ and CIE $y(r=0.25$, $P<0.05)$ and strongly correlated with CIE Y\% $(r=0.63, P<0.001)$. The inclusion in the mean intensity of minimal peaks such as A5 and B1 in Figure 3 may have been the problem because maximum peak intensity had a stronger relationship with CIE $x(r=0.39, P<0.1)$. Likewise, the mean intensity of interference peaks had little relationship to the 
distance from central white $(r=0.07, \mathrm{NS})$ whereas the intensities of the first, second and third largest peaks showed stronger relationships (respectively: $r=0.25, P<0.05 ; r=-0.45, P<0.001$; and $r=-0.42, P<0.001$ ). Note the change in sign of the correlation coefficients, this was because the largest peak was indicative of strong metallic spectra (Figure 3 , B) while second and third peaks were indicative of spectra with multiple peaks (Figure 3, A). In other words, a spectrum from a metallic myofiber with a single strong peak increased the distance from central white (as in the examples shown in Figure 4), while spectra with multiple peaks converged on the central white.

Another way to answer the question asked in the introduction was to take spectra with CIE \% $<10$ and to compare them with spectra with $\mathrm{CIE} \% \mathrm{Y}>20$, in other words, to compare myofibers with low scattering to myofibers with high scattering. Myofibers with low scattering had fewer interference peaks $(2.9 \pm 0.3, n=10)$ than myofibers with high scattering $(4.9 \pm 1.3, n=31, P<0.001)$. Thus, myofibers with high scattering (high CIE \%Y) had the most interference peaks so that the signal processing algorithm produced one parameter (number of peaks) acting in the opposite direction to all the other parameters. Excluding the number of peaks from a multivariate analysis, CIE \% Y was correlated with peak 3 intensity and mean peak width $(\mathrm{R}=0.81)$. In other words, spectra similar to that in Figure 3A (many wide peaks) were indicative of high scattering.

\section{REFERENCES}

[1] Swatland, H.J. (2013). Reflectance versus transmittance: the effects of light scattering on red colorants (carmine, Amazonian red annatto, and Peruvian cochinilla rojo and rosado) in biological, textile and museum science. Color Res. Appl. 39: 599-606. https://onlinelibrary.wiley.com/doi/abs/10.1002/col.21838

[2] Elliott, R.J. (1967). Effect of optical systems and sample preparation on the visible reflection spectra of pork muscle.

J. Sci. Food Agric. 18: 332-338. https://onlinelibrary.wiley.com/doi/abs/10.1002/jsfa.2740180802

[3] Irving, T.C., Swatland, H.J., Millman, B.M. (1989). Effect of pH on myofilament spacing in pork measured by x-ray diffraction. Can. Inst. Food Sci. Technol. J. 23: 79-81.https://www.researchgate.net/profile/Thomas_Irving/3

[4] Swatland, H.J. (1989) . Birefringence of beef and pork muscle fibers measured by scanning and ellipsometry with a computer-assisted polarising microscope. J. Comput. Assist. Microsc. 1: $249-262$. https://www.researchgate.net/publication/292002903_Birefringence_of_beef_and_pork_muscle_fibers_measured_by_ scanning_and_ellipsometry_with_a_computer-assisted_polarizing_microscope

[5] Swatland, H.J. (2012). Iridescence in beef caused by multilayer interference from sarcomere discs. Meat Sci. 90: 398401. https://www.sciencedirect.com/science/article/abs/pii/S0309174011002919

[6] Billmeyer, F.W. , Saltzman, M. (1981). Principles of Color Technology. Wiley, New York.

[7] Swatland, H.J. (2018). Colourimetry of chromatic aberration. Queckett J. Microsc. 43: $307-314$. https://www.researchgate.net/publication/330638074_Colourimetry_of_chromatic_aberration

[8] Swatland, H.J. (1989). Relationships between surface and internal reflectance in pork. Can. Inst. Food Sci. Technol. J. 22:165-169. https://www.sciencedirect.com/science/article/pii/S0315546389703540 